\title{
Improving thermal stability and efficacy of BCNU in treating glioma cells using PAA-functionalized graphene oxide
}

This article was published in the following Dove Press journal:

International Journal of Nanomedicine

29 March 2012

Number of times this article has been viewed

\section{Yu-Jen Lu',2,\# \\ Hung-Wei Yangl,\# \\ Sheng-Che Hung ${ }^{3}$ \\ Chiung-Yin Huang ${ }^{2}$ \\ Shin-Ming $\mathrm{Li}^{4}$ \\ Chen-Chi M Ma ${ }^{4}$ \\ Pin-Yuan Chen ${ }^{2}$ \\ Hong-Chieh Tsai \\ Kuo-Chen Wei ${ }^{2}$ \\ Jyh-Ping Chen'}

'Department of Chemical and Materials Engineering, Chang Gung University, Kwei-San, Taoyuan, Taiwan;

${ }^{2}$ Department of Neurosurgery, Chang

Gung Memorial Hospital, Kwei-San,

Taoyuan, Taiwan; ${ }^{3}$ Department of

Radiology, Taipei Veterans General

Hospital, Taipei, Taiwan; ${ }^{4}$ Department

of Chemical Engineering, National

Tsing Hua University, Hsinchu, Taiwan

\#These authors contributed equally to this work
Correspondence: Jyh-Ping Chen Department of Chemical and Materials Engineering, Chang Gung University, Kwei-San, Taoyuan 333, Taiwan, ROC Tel +88632 I I 8800

Fax +88632118668

Email jpchen@mail.cgu.edu.tw

Kuo-Chen Wei

Department of Neurosurgery, Chang Gung Memorial Hospital, Kwei-San,

Taoyuan 333, Taiwan, ROC

Tel $+8863328 \quad 1200$

Fax +88633285818

Email kuochenwei@adm.cgmh.org.tw
Background: 1,3-bis(2-chloroethyl)-1-nitrosourea (BCNU), a commercial chemotherapeutic drug for treating malignant brain tumors, has poor thermal stability and a short half-life. Immobilization of BCNU on a nanocarrier might increase the thermal stability of BCNU and extend its half-life.

Methods: Nanosized graphene oxide (GO) could be modified by polyacrylic acid (PAA) to improve the aqueous solubility and increase the cell penetration efficacy of the nanocarrier. PAA-GO intended as a drug carrier for BCNU was prepared and characterized in this study. The size and thickness of PAA-GO was investigated by transmission electron microscopy and atomic force microscopy, and the presence of PAA functional groups was confirmed by electron spectroscopy for chemical analysis and thermogravimetric analysis. BCNU was conjugated to PAA-GO by covalent binding for specific killing of cancer cells, which could also enhance the thermal stability of the drug.

Results: Single layer PAA-GO (about $1.9 \mathrm{~nm}$ ) with a lateral width as small as $36 \mathrm{~nm}$ was successfully prepared. The optimum drug immobilization condition was by reacting $0.5 \mathrm{mg}$ PAA-GO with $0.4 \mathrm{mg}$ BCNU, and the drug-loading capacity and residual drug activity were $198 \mu \mathrm{g}$ BCNU/mg PAA-GO and 70\%, respectively. This nanocarrier significantly prolonged the half-life of bound BCNU from 19 to 43 hours compared with free drug and showed efficient intracellular uptake by GL261 cancer cells. The in vitro anticancer efficacy of PAA-GO-BCNU was demonstrated by a $30 \%$ increase in DNA interstrand cross-linking and a $77 \%$ decrease in the $\mathrm{IC}_{50}$ value toward GL261 compared with the same dosage of free drug.

Conclusion: Nanosized PAA-GO serves as an efficient BCNU nanocarrier by covalent binding. This nanocarrier will be a promising new vehicle for an advanced drug delivery system in cancer therapy.

Keywords: graphene oxide, BCNU, glioma cells, drug delivery, thermal stability

\section{Introduction}

In recent years, advanced drug-delivery systems have improved cancer therapy outcomes with great promise. ${ }^{1}$ Development of different nanoscale drug carriers has been an important focus in advanced drug-delivery systems. ${ }^{2-4}$ Some recently approved advanced drug-delivery systems, like liposomes and/or polymer nanoparticles, have led to many obsolete drugs regaining the possibility of clinical use. ${ }^{5}$ An ideal advanced drug-delivery system includes controlled release (triggered release of drug under a characteristic environment of diseased cells) and drug protection until entering diseased cells, which aim to prevent early drug degradation and to increase the intracellular drug concentration. ${ }^{6}$ By using this system for drug delivery, we can not only improve treatment efficacy, but also decrease systemic toxicity. 
Since 2004, "graphene", a novel two-dimensional honeycomb material, has been recognized as one of the most promising materials used as a filler in polymer matrices. Graphene can enhance the mechanical strength, conductivity, and gas barrier performance of composite materials due to its high intrinsic mobility, high thermal conductivity, mechanical stiffness, excellent electrical conductivity, and good gas barrier properties. ${ }^{7,8}$ This versatile material has also found many applications in biological systems, such as drug delivery, gene delivery, and biosensors in the past two years. ${ }^{9-11}$ Compared with other carrier materials used in advanced drug-delivery systems, graphene oxide (GO) offers a high surface to weight ratio, high possibility for surface modification, high drug-loading efficiency, a $\mathrm{pH}$-responsive drug-delivery mechanism, and photothermal effects. ${ }^{12,13}$ Furthermore, it has been demonstrated that functionalized GO can enter mammalian cells, which renders it a reasonable drug carrier. ${ }^{9}$ Unlike the common poor water dispersion problem faced by other carbon nanomaterials, like fullerenes and carbon nanotubes, pristine GO has good aqueous solubility because of abundant hydrophilic groups on both sides of its large surface. The hydrophilic groups include hydroxyl, epoxide, and carboxylic groups. These functional groups help to maintain stability in physiological solutions and increase the ability to carry drugs. ${ }^{14}$

Brain malignancy is notorious for its high recurrence and mortality rate, although its prevalence is relatively low compared with other forms of cancer. Most malignant brain tumors in adults are glioblastoma multiforme (GBM). ${ }^{15}$ GBM is a highly invasive tumor originating from glial cells with indistinct borders along the normal parenchyma, and is difficult to remove by surgical excision. The median survival of GBM patients is approximately one year. ${ }^{16}$ The dismal prognosis and limited treatment choices give rise to the need for a novel and more efficient delivery route for chemotherapy against GBM. 1,3-bis(2-chloroethyl)1-nitrosourea (BCNU) is a commercial chemotherapeutic drug for brain malignancy because of its ability to penetrate the blood-brain barrier. The limitation to the clinical use of BCNU is its severe side effects, including bone marrow suppression, hepatotoxicity, and pulmonary fibrosis. ${ }^{17}$ In addition, metabolism in the liver after intravenous injection leads to transformation of BCNU into an active form before entering the brain tumor. To increase its usability, new forms of advanced drug-delivery systems have been developed for $\mathrm{BCNU}$ over the years. The most widely used delivery route in clinical practice is a BCNU wafer put on the tumor bed after surgery. Through gradual degradation of the matrix polymer,
$\mathrm{BCNU}$ is released into the peripheral brain parenchyma up to $2 \mathrm{~cm}$ in depth. ${ }^{18}$

Currently, all the methods used to bind drugs on GO are based on noncovalent interactions. For example, $\pi-\pi$ stacking prevails between $\mathrm{GO}$ and chemotherapeutic drugs possessing aromatic ring structures, such as doxorubicin, camptothecin, and SN-38. This adsorption binding method provides GO with a high drug-loading efficiency due to its high surface/ volume ratio for drug loading. ${ }^{19}$ Dai et al explained that PEGylated GO can carry water-insoluble cancer drugs such as camptothecin and SN38 and showed pH-dependent drug release behavior. ${ }^{9}$ Chen et al used a chemical coprecipitation method to add $\mathrm{Fe}_{3} \mathrm{O}_{4}$ magnetic nanoparticles onto graphene. After decorating with folic acid as a targeting ligand, the drug-loaded graphene selectively killed breast cancer cells with released doxorubicin. ${ }^{12}$ Tan et al modified graphene with polyvinyl alcohol for adsorption of doxorubicin to treat breast cancers cells. ${ }^{20}$ Zhang et al increased $\mathrm{pH}$-responsive drug-releasing ability by adding sulfonic acid on graphene. GO carried more than $40 \%$ doxorubicin and $4.5 \%$ camptothecin in weight ratio and released $35 \%$ doxorubicin and $17 \%$ camptothecin at $\mathrm{pH}$ 5.0. ${ }^{21}$ In another study, Zhang et al added polyethylenimine onto graphene to adsorb SiRNA. The positive charged polyethylenimine could attract SiRNA which is abundant with negative charged moiety. The drug resistance rate decreased through inhibited production of Bcl-2 protein by SiRNA and the cytotoxic efficacy of the doxorubicin on graphene increased. ${ }^{22}$ Similarly, Gan et al adsorbed plasmid DNA on chitosan-decorated graphene to increase the cytotoxic effect of camptothecin by DNA integration. ${ }^{23}$

As shown from previous discussion of up-to-date investigations using $\mathrm{GO}$ as a nanocarrier for anticancer drugs, the most popular drug-loading method is noncovalent binding. However, for an anticancer drug without aromatic ring structures, such as BCNU, $\pi-\pi$ stacking force will be weak and the most suitable method for drug loading may be covalent binding. To date, there have been no articles describing this alternative drug-loading method for $\mathrm{GO}$, which will widen the application of GO in drug or gene delivery. In this work, we first modified the GO surface with abundant polyacrylic acid (PAA) chains. PAA is a biocompatible material showing mucus-adhesive characteristics, which can enhance the solubility and dispersibility of GO in biological solutions without decreasing its high drug-loading ability. ${ }^{24}$ The carboxyl groups of PAA can react with BCNU through carbodiimidemediated amide bond formation to increase drug loading (Figure 1). To our best knowledge, this is the first paper describing covalent binding of a chemotherapeutic agent 


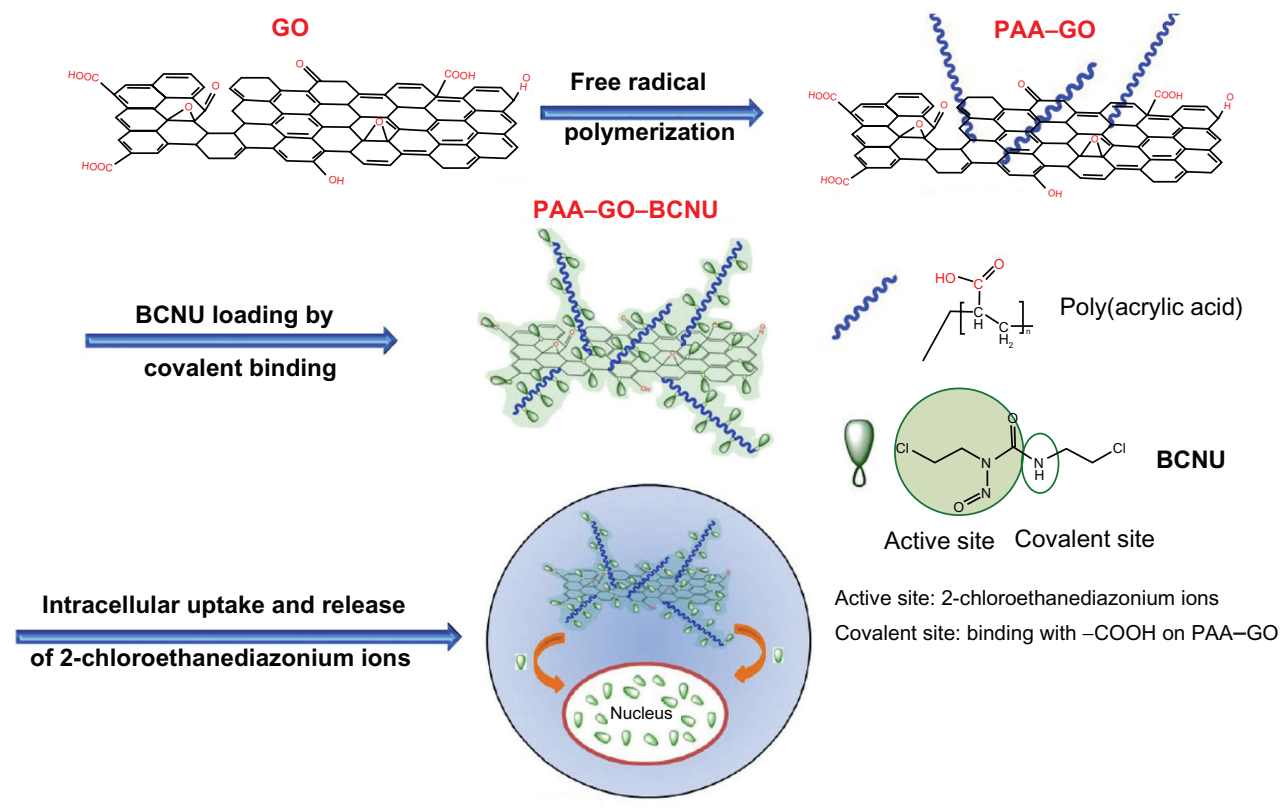

Figure I A schematic diagram showing synthesis of PAA-GO from GO, conjugation of BCNU to PAA-GO by covalent binding, delivery of PAA-GO-BCNU to cancer cells, and the cytotoxic effect of BCNU.

Abbreviations: GO, graphene oxide; PAA, polyacrylic acid; BCNU, I,3-bis(2-chloroethyl)-I-nitrosourea.

without aromatic structures. The results revealed excellent $\mathrm{IC}_{50}$ in killing malignant GL261 glioma cells. The thermal stability of PAA-GO-BCNU and cell apoptosis through an interstrand DNA cross-linking mechanism to validate this novel drug carrier was also studied.

\section{Materials and methods Materials}

The raw materials of graphite platelet (model xGnP) measuring $100 \mu \mathrm{m}$ in width and $5-15 \mathrm{~nm}$ in thickness were obtained from XG Sciences Inc (East Lansing, MI). Acrylic acid, potassium persulfate, sulfuric acid $\left(\mathrm{H}_{2} \mathrm{SO}_{4}, 98 \%\right)$, sodium sulfate $\left(\mathrm{Na}_{2} \mathrm{SO}_{4}\right)$, potassium permanganate $\left(\mathrm{KMnO}_{4}\right)$, hydrogen peroxide solution $\left(\mathrm{H}_{2} \mathrm{O}_{2}\right)$, and ammonia solution were purchased from Showa Chemical Co (Tokyo, Japan). BCNU was obtained from Boehringer Ingelheim (Mannheim, Germany). GL261 glioma cells were obtained from Professor Chia-Rui Shen at the Graduate Institute of Medical Biotechnology (Chang Gung University, Taiwan). Hank's balanced salt solution and RPMI-1640 were bought from Gibco (Grand Island, NY). Fetal bovine serum was bought from Biological Industries (Beit-Haemek, Israel). Gentamicin, penicillin, and streptomycin were obtained from MDBio. A LIVE/DEAD viability/cytotoxicity kit and minimum essential medium were bought from Invitrogen (Carlsbad, CA). Glutaraldehyde, 1-ethyl-3-(3-dimethylaminepropyl) carbodiimide hydrochloride (EDC), 2-( $N$-morpholino)ethanesulfonic acid hydrate
(MES), sulfanilamide, and $N$-hydroxysulfosuccinimide sodium salt (sulfo-NHS) were purchased from Sigma (St Louis, MO). Deionized water was used in all experiments. All reagents were of analytical grade and used without any further purification.

\section{Preparation of GO}

Preparation of GO followed the modified Hummers' method. ${ }^{25}$ Two hundred fifty milligrams of graphite platelet, $0.125 \mathrm{~g} \mathrm{NaNO}_{3}$, and $12 \mathrm{~mL}$ of $\mathrm{H}_{2} \mathrm{SO}_{4}(98 \%)$ were added into a $100 \mathrm{~mL}$ flask in an ice bath under magnetic stirring. Next, $0.75 \mathrm{~g} \mathrm{KMnO}_{4}$ was added slowly to the solution while keeping the temperature below $5^{\circ} \mathrm{C}$. After adding $\mathrm{KMnO}_{4}$, the ice bath was removed and the flask was heated to $100^{\circ} \mathrm{C}$, followed by slow addition of $12 \mathrm{~mL}$ of deionized water. The temperature of the solution was increased to $98^{\circ} \mathrm{C}$ and the solution was maintained at this temperature for 30 minutes. Next, $50 \mathrm{~mL}$ of deionized water with $10 \% \mathrm{H}_{2} \mathrm{O}_{2}$ was added to the solution until the cessation of gas evolution. The solution was centrifuged at $11,000 \mathrm{rpm}$ and washed using deionized water several times to remove impurities. The precipitate was collected and subject to ultrasonication for 8 hours at $800 \mathrm{~W}$ using an Ultrasonic Liquid Processor 2020 from Misonix (Farmingdale, NY) and centrifugation at $10,000 \mathrm{rpm}$ for 3 hours. The supernatant was collected and filtered with Acrodisc $25 \mathrm{~mm}$ syringe filters ( $0.2 \mu \mathrm{m}$ Supor membrane) three times, and the GO collected in the filtrate was subjected to further modification. 


\section{Preparation of PAA-NGO}

PAA-grafted GO (PAA-GO) was synthesized by free radical polymerization. In a typical experiment, $0.1 \mathrm{~g} \mathrm{GO}$ and $40 \mathrm{~mL}$ $\mathrm{H}_{2} \mathrm{O}$ were added into a $100 \mathrm{~mL}$ four-necked flask. After low-energy ultrasonication ( $12 \mathrm{~W}, 55 \mathrm{kHz}, 30$ minutes), the flask was stirred in a thermostatic oil bath at $50^{\circ} \mathrm{C}$ under a nitrogen atmosphere. Then, a well-mixed solution of $15 \mathrm{mg}$ potassium persulfate dissolved in $2 \mathrm{~g}$ acrylic acid monomers was added through a tube pumped at a rate of $0.5 \mathrm{~mL} /$ minute. The free radical polymerization reaction was initiated by potassium persulfate, which was continued for 8 hours. After polymerization, the solution was centrifuged at $11,000 \mathrm{rpm}$ and washed using deionized water several times to remove the impurities and reactants. The above steps were repeated 5-6 times to remove unreacted acrylic acid completely and the product was dried in a vacuum oven at $80^{\circ} \mathrm{C}$ overnight to remove the solvent, after which PAA-GO in the form of a black powder was obtained.

\section{Characterization of GO and PAA-GO}

An atomic force microscope (XE-100; PISA, Santa Clara, CA) was used to analyze the surface topography and size of the nanocarrier. Diluted GO and PAA-GO in alcohol were deposited onto a freshly cleaved mica substrate and imaged after alcohol evaporation. Experiments were performed with a tapping mode and all images were recorded in air at room temperature at 256 pixels, with a scan size of $0.5 \times 0.5 \mu \mathrm{m}^{2}$ and a scan speed of $0.9 \mathrm{~Hz}$. GO and PAA-GO were subject to $\mathrm{X}$-ray photoelectron spectroscopy analysis using a ESCALAB 220 iXL (VG Scientific, East Grinstead, UK) spectrometer equipped with a hemispherical electron analyzer and a $\mathrm{Mg} \mathrm{K}$ $(\mathrm{hv}=1487.7 \mathrm{eV}) \mathrm{X}$-ray source. A small spot lens system was needed to analyze a sample less than $1 \mathrm{~mm}^{2}$ in area. The PAA content of PAA-GO was determined by thermogravimetric analysis using an STA 449 PC from Netzsch. Analysis was performed with $20 \mathrm{mg}$ of samples in a platinum pan under nitrogen atmosphere. The nominal gas flow was $5 \mathrm{~mL} /$ minute, and the heating velocity was $20^{\circ} \mathrm{C} /$ minute.

\section{Immobilization of BCNU on PAA-GO}

The BCNU was dissolved in a 70:30 (v/v) mixture of deionized water and ethanol with a concentration of $10 \mathrm{mg} / \mathrm{mL}$. A $0.1 \mathrm{~mL}$ aliquot of EDC solution $(12 \mathrm{mg} / \mathrm{mL}$ EDC in $0.5 \mathrm{M}$ MES buffer, $\mathrm{pH}$ 6) was mixed with $0.1 \mathrm{~mL}$ of PAA-GO $\left(5 \mathrm{mg} / \mathrm{mL}\right.$ ) at $25^{\circ} \mathrm{C}$ and sonicated for 20 minutes in the dark at room temperature. After separation with centrifugation at $7000 \mathrm{rpm}$ for $7 \mathrm{~min}, \mathrm{PAA}-\mathrm{GO}$ was washed twice using $0.5 \mathrm{~mL}$ of deionized water, resuspended in $0.2 \mathrm{~mL}$ of $0.1 \mathrm{M}$
MES buffer, and mixed with $0.2 \mathrm{~mL}$ BCNU of different concentrations at $10^{\circ} \mathrm{C}$. The solutions were mixed by sonication for 0.5 hours and then shaken for another 1.5 hours at $10^{\circ} \mathrm{C}$. PAA-GO with BCNU immobilized on the surface were separated from the solution using centrifugation, washed with pure alcohol and deionized water, washed again with deionized water to remove residual alcohol, and dispersed in $0.1 \mathrm{~mL}$ of deionized water. ${ }^{26}$ To calculate the ratio of BCNU immobilized on PAA-GO (ie, PAA-GO-BCNU), the supernatant was analyzed by high-performance liquid chromatography on a SUPELCOSIL LC-18 column (4.6 $\times 250 \mathrm{~mm})$ using a L-2130 pump and a L-2400 ultraviolet detector (Hitachi, Tokyo, Japan). The mobile phase was a 40:60 (v/v) mixture of deionized water and methanol with a flow rate of $2 \mathrm{~mL} /$ minute. The absorbance data were obtained at $270 \mathrm{~nm}$.

\section{Thermal stability study of BCNU}

The stabilities of free $\mathrm{BCNU}$ and $\mathrm{PAA}-\mathrm{GO}-\mathrm{BCNU}$ at $1 \mathrm{mg} / \mathrm{mL} \mathrm{BCNU}$ were tested by incubation at $37^{\circ} \mathrm{C}$ for up to 14 days. The drug activities were analyzed using the Bratton-Marshall assay. ${ }^{27,28}$ Forty microliters of free BCNU and PAA-GO-BCNU solution were reacted with $80 \mu \mathrm{L}$ of sulfanilamide solution $(5 \mathrm{mg} / \mathrm{mL}$ in $2 \mathrm{M} \mathrm{HCl})$ at $50^{\circ} \mathrm{C}$ for 45 minutes and then cooled to room temperature. One hundred microliters of the above solution and $10 \mu \mathrm{L}$ of Bratton-Marshall reagent ( $3 \mathrm{mg} / \mathrm{mL}$ of $\mathrm{N}$-(1-naphthyl) ethylenediamine in deionized water) were reacted for one minute and absorbances were measured at $540 \mathrm{~nm}$ using a Genesys 20 spectrophotometer (Thermo Scientific, Barrington IL).

\section{Cellular uptake study}

To prepare fluorescent PAA-GO, EDC $12 \mathrm{mg}$ and sulfo-NHS $27 \mathrm{mg}$ were dissolved in $2 \mathrm{~mL}$ of $0.5 \mathrm{M}$ MES buffer ( $\mathrm{pH}$ 6.3) in the dark. A $0.2 \mathrm{~mL}$ aliquot of the above solution was mixed with $0.2 \mathrm{~mL}$ of PAA-GO $(5 \mathrm{mg} / \mathrm{mL})$ at $25^{\circ} \mathrm{C}$ and reacted for 30 minutes in the dark. After separation and washing with deionized water, activated PAA-GO was added to $0.2 \mathrm{~mL}$ of MES buffer, mixed with $0.1 \mathrm{~mL}$ of the monofunctional hydrazide cyanine $5(\mathrm{Cy} 5)$ at $25^{\circ} \mathrm{C}$, and then vortexed for 2 hours in the dark. The PAA-GO with Cy5 immobilized on the surface (PAA-GO-Cy5) were separated from the solution and washed with deionized water. GL261 cells were cultured in RPMI-1640 supplemented with $2.2 \mathrm{mg} / \mathrm{mL}$ sodium carbonate, $10 \%$ fetal bovine serum, $50 \mu \mathrm{g} / \mathrm{mL}$ gentamicin, $50 \mu \mathrm{g} / \mathrm{mL}$ penicillin, and $50 \mu \mathrm{g} / \mathrm{mL}$ streptomycin at $37^{\circ} \mathrm{C}$ and $5 \% \mathrm{CO}_{2}$. Approximately $2 \mathrm{~mL}$ of GL261 cells $(10,000$ cells $/ \mathrm{mL})$ were plated in $35 \mathrm{~mm}$ diameter plates and 
cultured in a humidified chamber at $37^{\circ} \mathrm{C}$ and $5 \% \mathrm{CO}_{2}$ for 48 hours. PAA-GO-Cy5 in $100 \mu \mathrm{L}$ of minimum essential medium were added, and the incubation was continued for 6 hours. The medium was removed, and the cells were washed with $1 \mathrm{~mL}$ of Hank's balanced salt solution and then fixed with fresh ethanol on ice for 5 minutes at room temperature. The cells were washed three times with Hank's balanced salt solution and analyzed on a TCS SP2 confocal microscope (Lecia, Wetzlar, Germany).

For transmission electron microscopic observation, GL261 cells were grown to $50 \%-60 \%$ confluence on Thermonax coverslips and treated with PAA-GO for 2 hours, followed by washes in phosphate-buffered saline and additional incubation in fresh media for 1 hour. Next, the cells were fixed in a mixture of $2.5 \%$ paraformaldehyde and $2.0 \%$ glutaraldehyde in phosphate-buffered saline for 30 minutes followed by extensive washes in phosphate-buffered saline. Then the cells were rinsed in $0.1 \mathrm{M}$ sodium cacodylate buffer ( $\mathrm{pH} \mathrm{7.4)}$ and postfixed in 1.0\% osmium tetroxide in the above buffer for 30 minutes. After several rinses in the sodium cacodylate buffer, the samples were dehydrated in a series of ethanol solutions $(20 \%, 40 \%, 60 \%, 75 \%$, and $95 \%$ for 5 minutes and $100 \%$ for 30 minutes with three changes) and infiltrated with Epon-Araldite (Ted Pella, Redding, CA) for 2 days (25\% Epon-Araldite and ethanol for 2 hours, $50 \%$ for 4 hours, $75 \%$ for overnight, and $100 \%$ for 1 day, with two changes). Samples were polymerized at $60^{\circ} \mathrm{C}$ for 2 days. Next, ultrathin sections (approximately $80 \mathrm{~nm}$ ) were cut on a Reichert Ultracut E Microtome (American Optical, Buffalo, NY) and collected on film-supported slot grids. Sections were lightly counterstained with uranyl acetate and lead citrate, and examined under a FEI CM120 transmission electron microscope equipped with a Gatan GIF100 image filter and operated at a beam energy of $120 \mathrm{keV}$.

\section{DNA cross-linking test}

The ethidium bromide fluorescence assay ${ }^{29}$ was used to measure the level of DNA cross-linking caused by BCNU in GL261 cells. The cells were exposed initially to different concentrations $(5-30 \mu \mathrm{g} / \mathrm{mL})$ of free BCNU or PAA-GO-BCNU and incubated for 12 hours. After incubation, about $1 \times 10^{6}$ cells were collected by centrifugation at $5000 \mathrm{rpm}$ for 5 minutes at $8^{\circ} \mathrm{C}$ and resuspended in phosphate-buffered saline. Forty microliters of the cell suspension were incubated for 15 minutes at $4^{\circ} \mathrm{C}$ with $200 \mu \mathrm{L}$ of lysis buffer. After lysis, the cell pellets were separated by centrifugation at $5000 \mathrm{rpm}$ for 6 minutes and the suspension was incubated with $25 \mu \mathrm{L}$ of heparin $(500 \mathrm{IU} / \mathrm{mL})$ for another 20 minutes at $37^{\circ} \mathrm{C}$, followed by addition of $1 \mathrm{~mL}$ of ethidium bromide solution. The mixture was heated for 5 minutes at $100^{\circ} \mathrm{C}$ to denature the DNA, then cooled in an ice bath for 6 minutes to renature it. Fluorescence was measured with excitation and emission wavelengths of $525 \mathrm{~nm}$ and $580 \mathrm{~nm}$, respectively.

\section{In vitro anticancer efficacy}

GL261 cells were cultured in RPMI-1640 supplemented with $2.2 \mathrm{mg} / \mathrm{mL}$ sodium carbonate, $10 \%$ fetal bovine serum, $50 \mu \mathrm{g} / \mathrm{mL}$ gentamicin, $50 \mu \mathrm{g} / \mathrm{mL}$ penicillin, and $50 \mu \mathrm{g} / \mathrm{mL}$ streptomycin at $37^{\circ} \mathrm{C}$ and $5 \% \mathrm{CO}_{2}$. Approximately 10,000 cells were placed in each well of a 96-well culture plate and incubated in a humidified chamber at $37^{\circ} \mathrm{C}$ and $5 \% \mathrm{CO}_{2}$ for 24 hours. Free BCNU and PAA-GO-BCNU (BCNU concentration $0-100 \mu \mathrm{g} / \mathrm{mL}$ ) were added to the medium and the culture continued. Cell growth was observed by counting the cells after 24 and 48 hours. Before counting, the culture medium was removed and cells were incubated in $120 \mu \mathrm{L}$ of XTT solution for 3 hours. After the reaction, $100 \mu \mathrm{L}$ of XTT solution was sampled from each well and transferred to a 96-well counting dish. Cytotoxicity toward GL261 cells in vitro was evaluated by measuring the optical density at $490 \mathrm{~nm}$ using an enzymelinked immunosorbent assay reader. In a separate series of experiments, $2 \mathrm{~mL}$ of GL261 cells (10,000 cells/mL) were plated in $35 \mathrm{~mm}$ diameter plates and cultured in a humidified chamber at $37^{\circ} \mathrm{C}$ and $5 \% \mathrm{CO}_{2}$ for 72 hours. Free BCNU and PAA-GO-BCNU (BCNU concentration 0, 2, 5, $10 \mu \mathrm{g} / \mathrm{mL}$ ) in $100 \mu \mathrm{L}$ of culture medium were added and the incubation continued for 12 hours. The medium was removed, the cells were washed with $1 \mathrm{~mL}$ of Hank's balanced salt solution, and $1 \mathrm{~mL}$ of LIVE/DEAD reagent was added. After 30 minutes, the reagent was removed and the cells were washed again with Hank's balanced salt solution. Cytotoxicity was monitored using a Leica TCS SP2 confocal microscope.

\section{Results and discussion}

\section{Characterization of PAA-GO}

The nanocarrier were prepared using a modified Hummer method and followed by long-term ultrasonication to obtain nanosized GO. After ultrasonication treatment, the lateral width of GO were shortened from several hundred nanometers to be less than $50 \mathrm{~nm}$, but still remained single-layered (about $1 \mathrm{~nm}$ ), as shown by atomic force microscopy images (Figure 2A). Next PAA was grafted onto GO to form PAANGO via free radical polymerization. The morphology of PAA-NGO observed by atomic force microscopy exhibited similar lateral widths with $\mathrm{GO}$, indicating that the dimension of GO and PAA-GO were almost the same. The thicknesses 

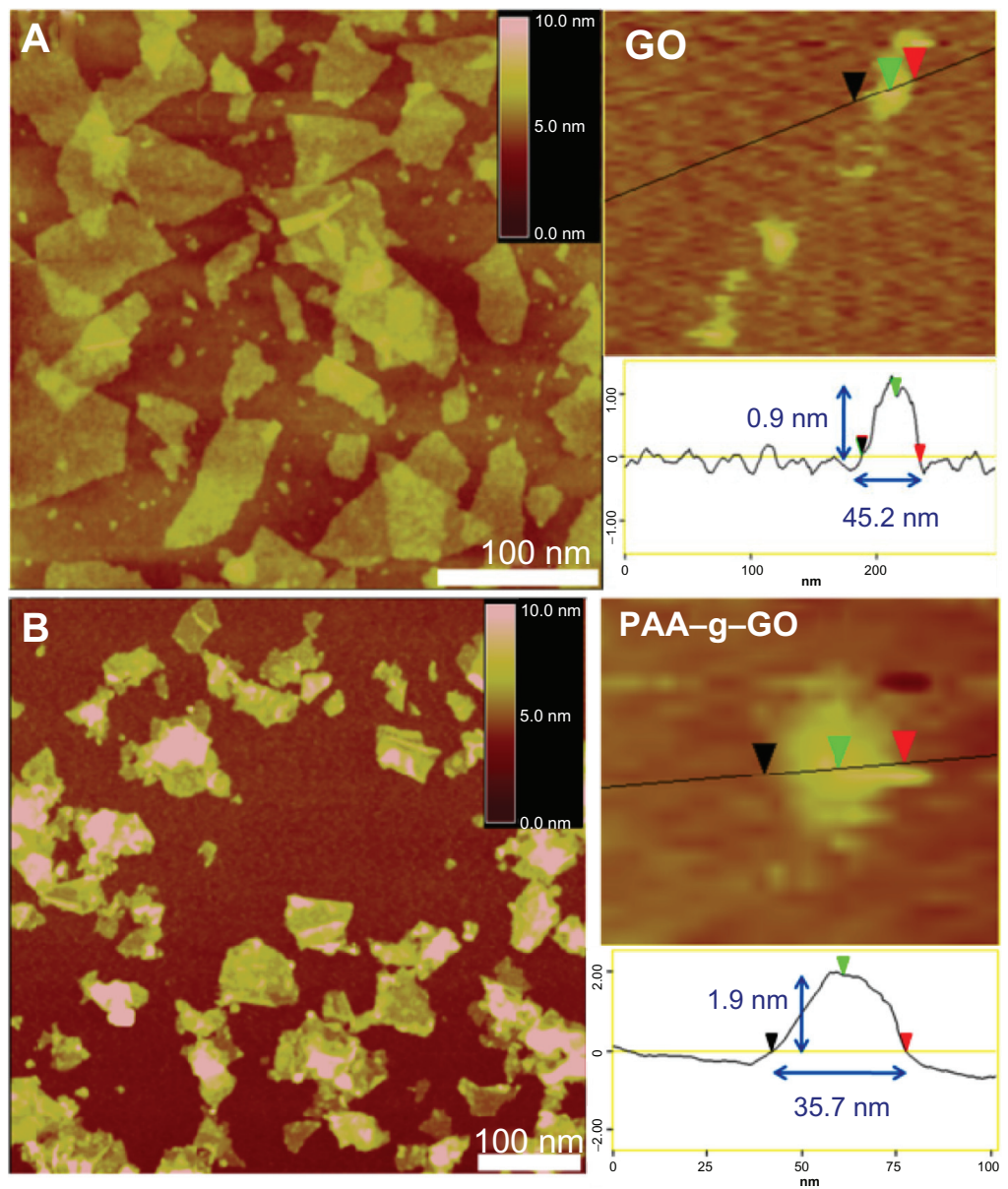

Figure 2 Atomic force microscopic images of (A) GO, and (B) PAA-GO. Abbreviations: GO, graphene oxide; PAA, polyacrylic acid.

of PAA-GO increased from $0.9 \mathrm{~nm}$ to $1.9 \mathrm{~nm}$ after grafting PAA chains onto the GO surface (Figure $2 \mathrm{~B}$ ). Controlling the dimensions of PAA-GO to within $2 \times 40 \mathrm{~nm}$ is very helpful for their efficient intracellular uptake into cells. ${ }^{11}$ Moreover, no contaminating particles appeared on the surface of both GO and PAA-GO, revealing great purity of these two materials. PAA-GO also showed excellent dispersibility and did not agglomerate after storage in water for several months.

Because different structural forms of carbon exhibit different oxidation behaviors, thermogravimetric analysis is a powerful tool for investigating the thermal stability of the graphene-based materials and the thermal stability of surface-functionalized GO materials. The compositions of GO and PAA-GO were studied by thermogravimetric analysis and the results are shown in Figure 3. The thermogravimetric analysis thermograms of both samples showed a small weight loss $(<5 \mathrm{wt} \%)$ at low temperature $\left(<200^{\circ} \mathrm{C}\right)$, which can be attributed to loss of absorbed water and decomposition of oxygen-containing functional groups. However, within the temperature range from $200^{\circ} \mathrm{C}$ to $250^{\circ} \mathrm{C}$, the thermogravimetric analysis curve of GO indicated a much smaller mass loss compared with PAA-GO, and PAA-GO showed a maximum weight loss at $235^{\circ} \mathrm{C}$ from the derivative weight loss curve (Figure 3). This phenomenon corresponded with the thermal decomposition of the PAA polymer backbone. When the temperature further increased $\left(>300^{\circ} \mathrm{C}\right)$, weight loss of both GO and PAA-GO could be attributed to the decomposition of GO. By calculating the weight loss percentage of PAA-GO from $200^{\circ} \mathrm{C}$ to $250^{\circ} \mathrm{C}$, it could be estimated that the content of PAA in PAA-NGO was about $16.7 \%$.

$\mathrm{X}$-ray photoelectron spectroscopy was used further to analyze the surface composition and structure of GO and PAA-GO by providing information of core-level binding energy, and to detect the functional groups present on PAA-GO based on chemical shifts. The $\mathrm{C} 1 \mathrm{~s}$ core level spectra of GO are presented in Figure 4A, which shows two major peaks $(284.0 \mathrm{eV}$ and $284.9 \mathrm{eV})$ attributing to the graphitic structure. ${ }^{30}$ Figure 4B shows additional peaks at higher binding energies for PAA-GO, indicating the presence of carbon atoms in other functional groups. The additional $\mathrm{X}$-ray photon 


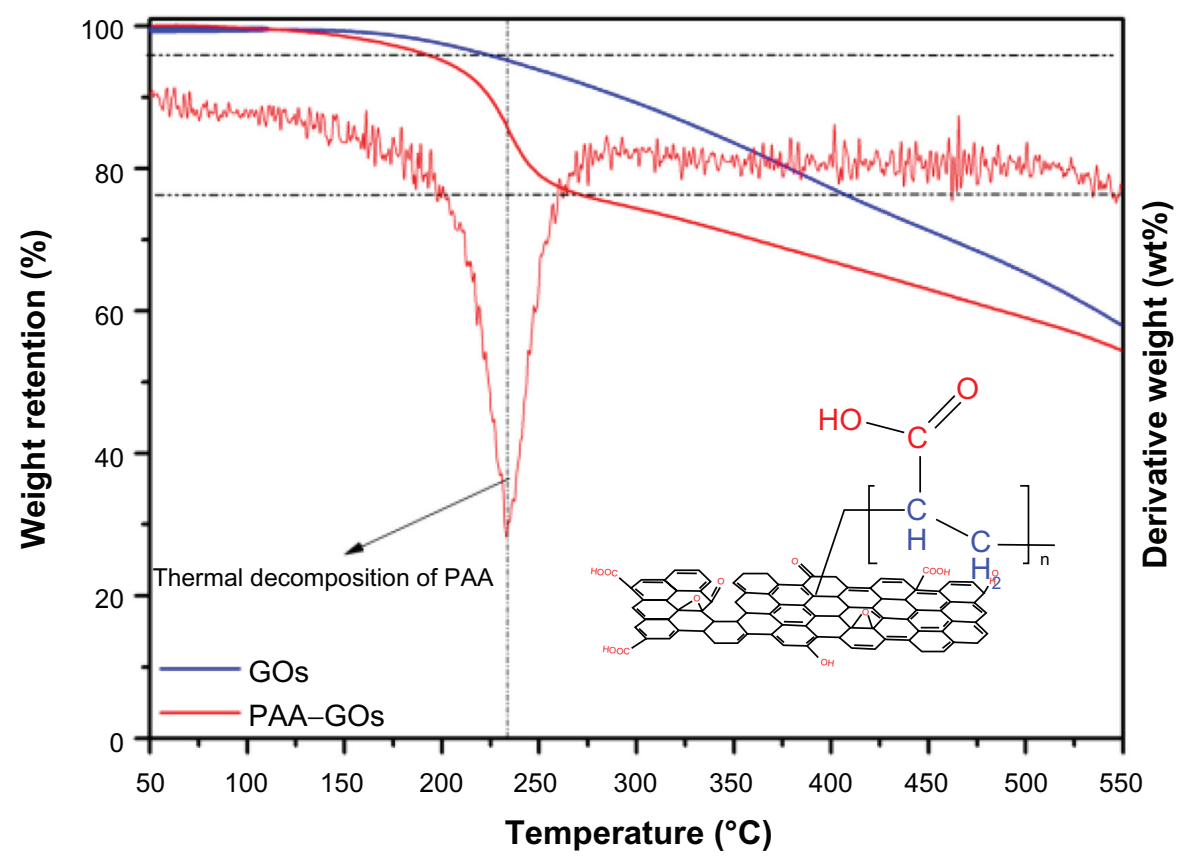

Figure 3 TGA curves of GO and PAA-GO.

Abbreviations: GO, graphene oxide; PAA, polyacrylic acid; TGA, thermogravimetric analysis.

spectroscopy peaks of PAA-GO, appearing at $285.1 \mathrm{eV}$ and 285.7, originate from the PAA main chain structure. ${ }^{31,32}$ The presence of these structure and functional group agrees well with the expected surface structure of PAA-GO. Furthermore, $\mathrm{x}$-ray photon spectroscopy results of PAA-GO show that the PAA main chain structure and the $-\mathrm{COOH}$ functional group have a 1:1 peak ratio, providing clear evidence that GO has been successfully grafted with PAA.

\section{Quantification and stability of PAA-GO-BCNU}

To be an effective therapy, BCNU must be efficiently immobilized on the surface of the PAA-GO to provide an effective therapeutic dose without requiring repeated injection of PAA-GO-BCNU, thus alleviating the side effects of BCNU. As shown in Figure 5A, the drug-loading capacity increased with the amount of BCNU used in the conjugation reaction, and reached a plateau when $\geq 0.4 \mathrm{mg}$ BCNU was used, with the maximum loading capacity being about $200 \mu \mathrm{g}$ BCNU/g PAA-GO. On the contrary, drug activity decreased with the amount of BCNU used in the conjugation reaction due to steric hindrance and/or structural changes, and leveled off at $65 \%$ when $\geq 0.4 \mathrm{mg}$ BCNU was used. Considering both drug-loading capacity and activity, $0.4 \mathrm{mg} \mathrm{BCNU}$ was deemed to be the optimum condition to react with $0.5 \mathrm{mg}$ PAA-GO for immobilization of BCNU, where the drug-loading capacity and activity are $198 \mu \mathrm{g}$ $\mathrm{BCNU} / \mathrm{mg}$ PAA-GO and $70 \%$, respectively.
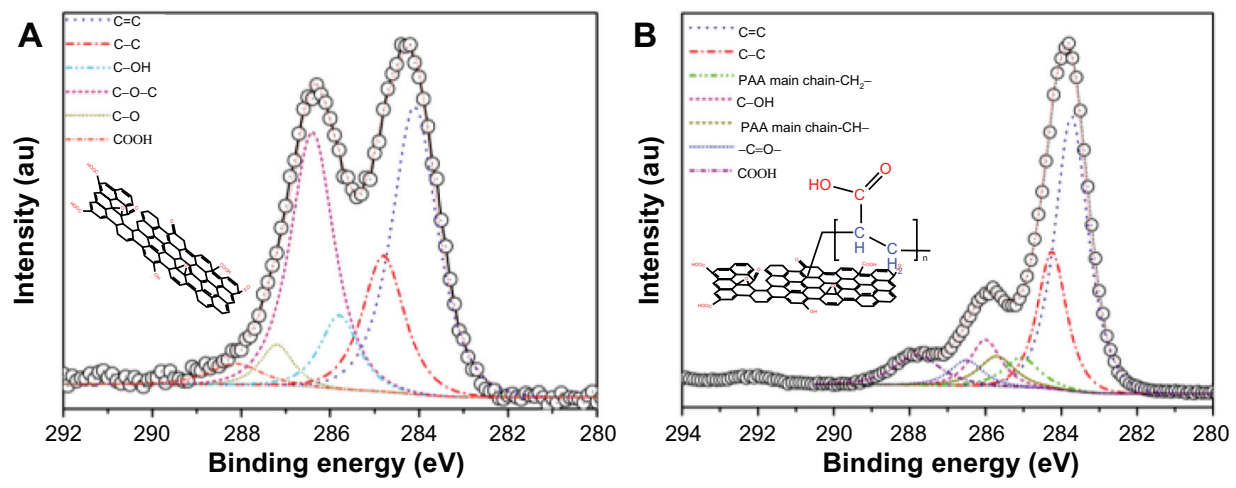

Figure 4 X-ray photoelectron spectroscopy of $\mathrm{Cl}$ s peaks of (A) GO, and (B) PAA-GO. Abbreviations: GO, graphene oxide; PAA, polyacrylic acid. 


\section{A}

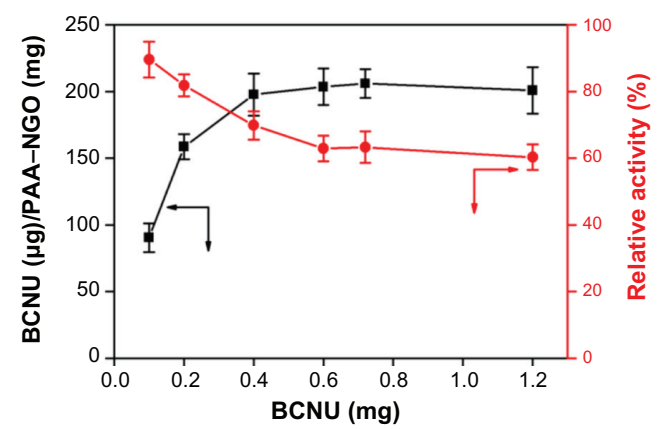

B

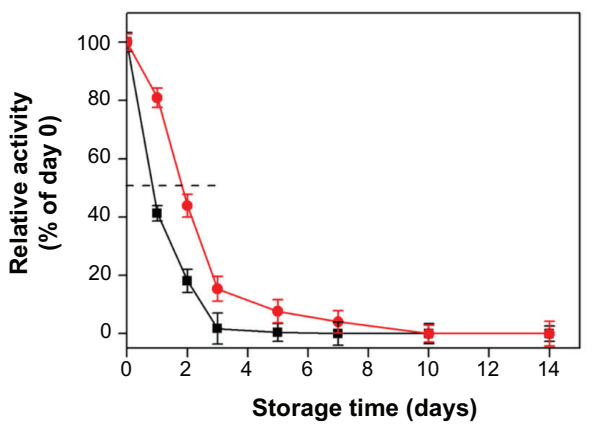

Figure 5 (A) Drug-loading capacity $(\mathbf{\square})$ and residual drug activity $(\bullet)$ of BCNU immobilized to PAA-GO. (B) Relative stability of free BCNU ( $\bullet$ ) and PAA-GO-BCNU $(\bullet)$ at $37^{\circ} \mathrm{C}$ where activities at day 0 were taken as $100 \%$ for free $\mathrm{BCNU}$ and PAA-GO-BCNU, respectively.

Note: Values are the mean \pm standard deviation $(n=6)$.

Abbreviations: GO, graphene oxide; PAA, polyacrylic acid; BCNU, I,3-bis(2-chloroethyl)-I-nitrosourea.

BCNU has poor thermal stability and a short half-life, and its hydrolysis rate was reported to increase with increasing temperature, resulting in a failure to crosslink DNA..$^{33}$ Immobilization of BCNU to a nanocarrier might offer additional advantages compared with free drug by increasing the thermal stability of BCNU. Indeed, the hydrolysis rate of BCNU was decreased after conjugation onto the surface of PAA-GO, which resulted in an increased half-life from
19 hours to 43 hours compared with free BCNU (Figure 5B). The improvement could have arisen from the immobilization of BCNU onto PAA-GO through covalent binding, which protects drug conformation, prevents distortion of its molecular structure, and then extends the half-life of drug activity. ${ }^{34}$ Therefore, PAA-GO is expected to protect BCNU better from nonspecific hydrolysis, which can preserve the release of active 2-chloroethanediazonium ions and the DNA
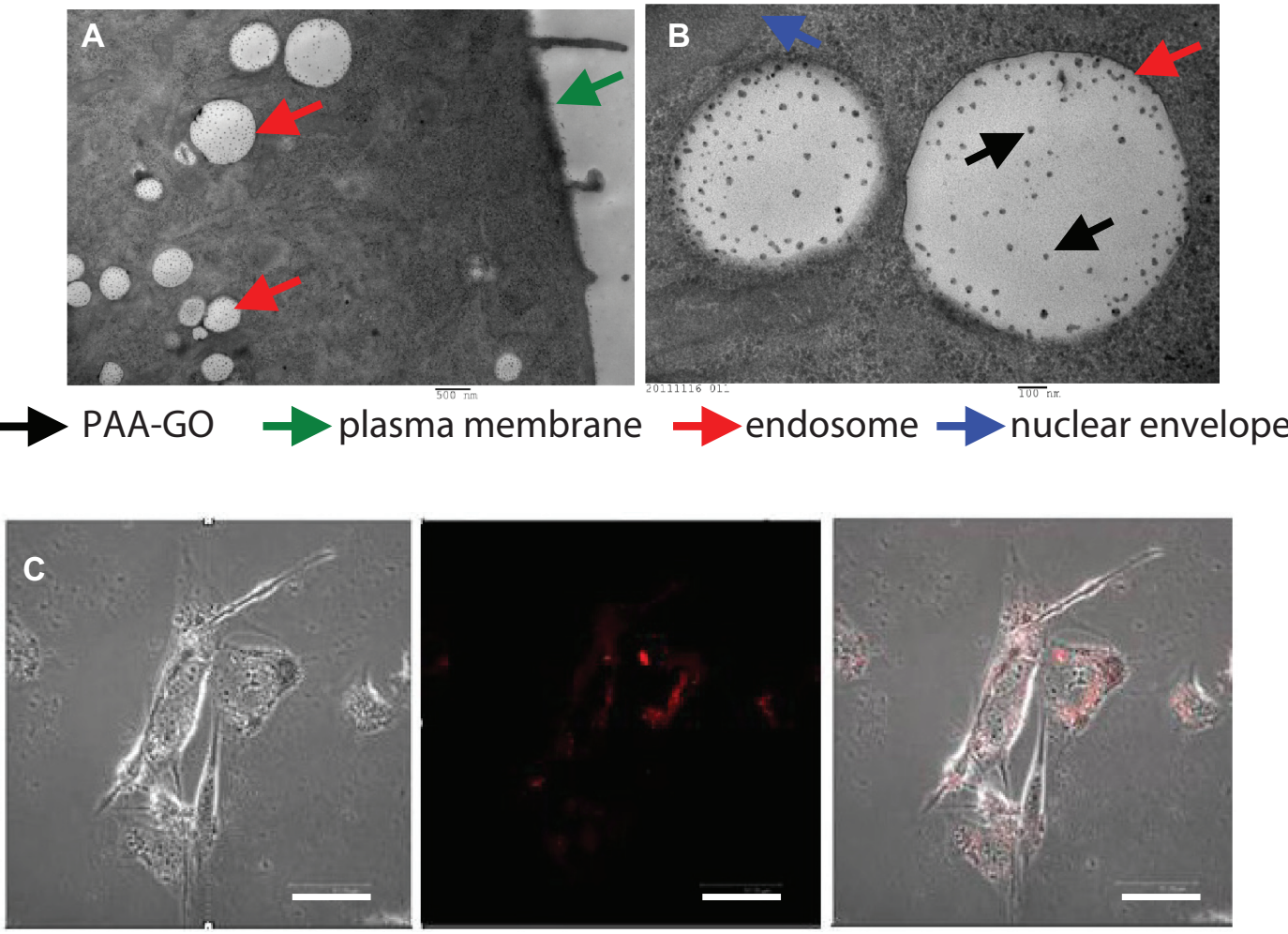

Figure 6 (A and B) Transmission electron micrographs of GL26I cells cocultured with PAA-GO. (C) Fluorescence micrographs of GL26I cells cultured with PAA-GO-Cy5. Notes: Phase image (left), fluorescence image (middle), merged image (right). (A) Bar $=500 \mathrm{~nm}$, (B) bar $=100 \mathrm{~nm},(\mathbf{C}) \mathrm{bar}=5 \mu \mathrm{m}$.

Abbreviations: GO, graphene oxide; PAA, polyacrylic acid. 


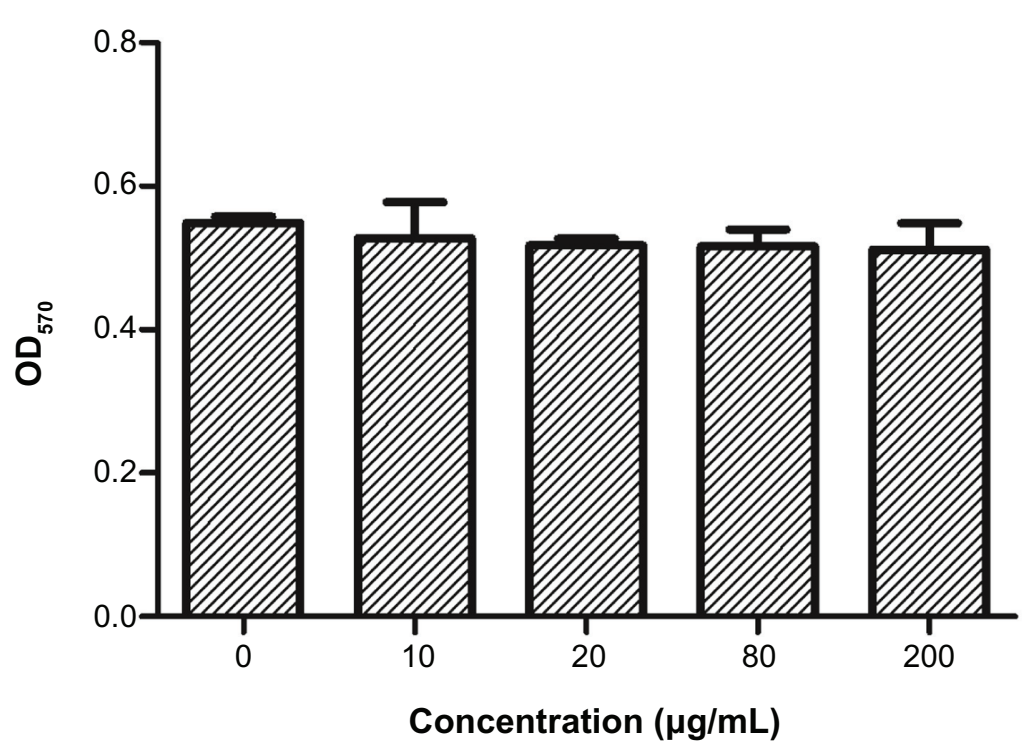

Figure 7 Viability of GL26I cells after incubation for 48 hours with PAA-GO $(0-200 \mu \mathrm{g} / \mathrm{mL})$ and assayed by the MTT method by measuring absorbance at $570 \mathrm{~nm}\left(\mathrm{OD}_{570}\right)$. Note: Values are the mean \pm standard deviation $(n=6)$.

Abbreviations: GO, graphene oxide; PAA, polyacrylic acid.

cross-linking ability of BCNU after intracellular uptake of PAA-GO-BCNU to ensure a higher effective drug dosage toward cancer cells (Figure 1).

\section{Cellular uptake of PAA-GO}

All eukaryotic cells continuously ingest parts of their plasma membranes via endocytosis. Endocytotic vesicles are generated when segments of the plasma membrane invaginate, enclosing extracellular substances. Most endocytotic vesicles fuse with primary lysosomes to form secondary lysosomes that contain hydrolytic enzymes and act as specialized organelles for intracellular disposal. ${ }^{35}$ Internalization of PAA-GO by GL261 cells was observed by transmission electron microscopy. Intracellular uptake was evident after contacting PAA-GO with cells for 6 hours, which are found within endosomes in the cytoplasmic region (Figure 6A). The presence of PAA-GO in close proximity to the nuclear region can be also observed (Figure 6B). The intracellular PAA-GO was also investigated by culture of GL261 cells with PAA-GO-Cy5 for 6 hours (Figure 6C). Indeed, PAA-GO (red color) could be detected within the cytoplasm of cells. In vitro confocal and transmission electron microscopic images therefore strongly support efficient entry of PAA-GO into cancer cells, which could potentially enhance the apoptotic effects of immobilized BCNU via efficient endocytic pathway and raise the effective intracellular drug activity. ${ }^{34}$

\section{In vitro anticancer efficacy}

The cytotoxicity of PAA-GO-BCNU toward GL261 cells was determined using the XTT assay. In control experiments,
A

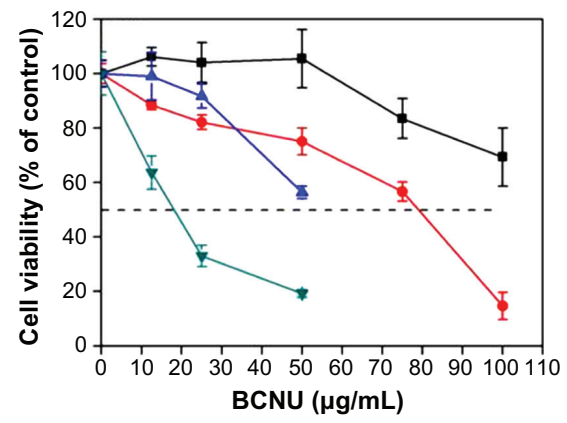

B

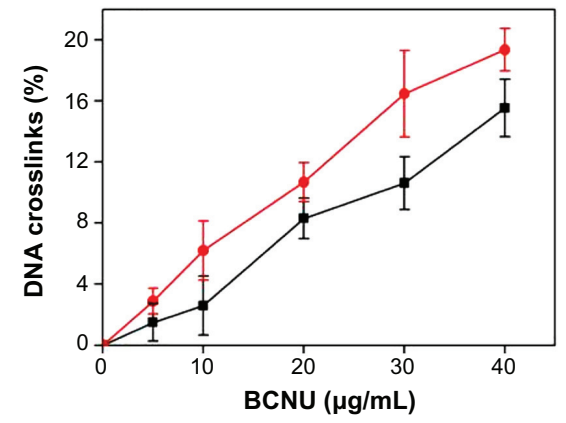

Figure 8 (A) Viability of GL26I cells after incubation for 24 hours with free BCNU (घ), PAA-GO-BCNU ( $\mathbf{\Delta}$ ); for 48 hours with free-BCNU (•), PAA-GO-BCNU ( $\mathbf{\nabla})$. (B) DNA interstrand crosslinking in GL26I cells after treatment 12 hours with free BCNU (๘) and PAA-GO-BCNU (•).

Note: Values are the mean \pm standard deviation $(n=5)$.

Abbreviations: GO, graphene oxide; PAA, polyacrylic acid; BCNU, I,3-bis(2-chloroethyl)-I-nitrosourea. 

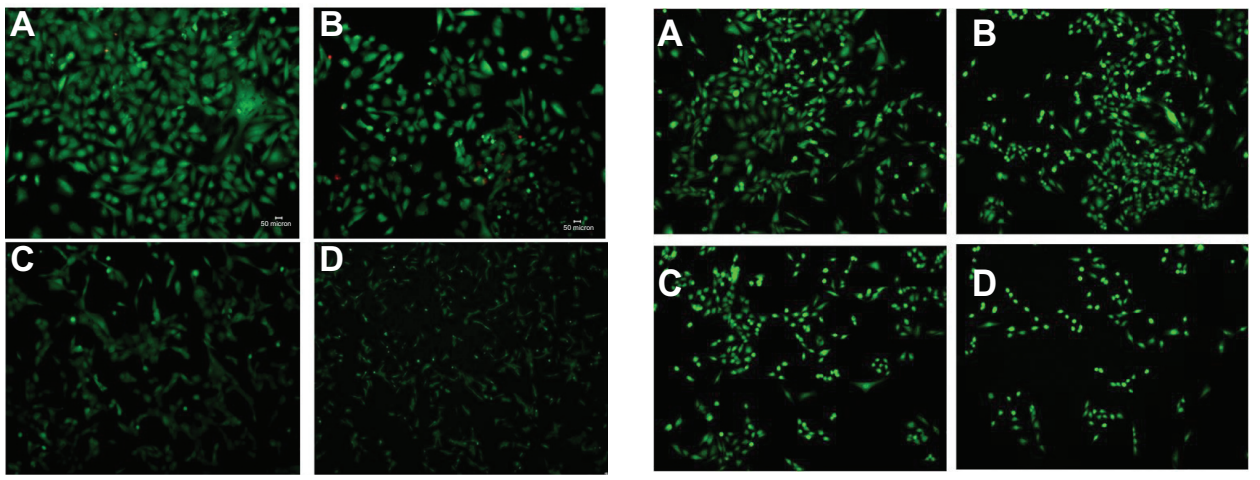

Figure 9 Fluorescence micrographs of GL26I cells after exposure to free BCNU (left) and PAA-GO-BCNU (right) for I2 hours and stained with a LIVE/DEAD viability/ cytotoxicity kit. BCNU concentrations: (A) $0 \mu \mathrm{g} / \mathrm{mL}$, (B) $2 \mu \mathrm{g} / \mathrm{mL}$, (C) $5 \mu \mathrm{g} / \mathrm{mL}$, and (D) $10 \mu \mathrm{g} / \mathrm{mL}$.

Notes: Red and green colors denote dead cells and green cells, respectively. The red color is hardly seen because dead cells easily detached from the plate after death. Abbreviations: GO, graphene oxide; PAA, polyacrylic acid; BCNU, I,3-bis(2-chloroethyl)-I-nitrosourea.

cells cultured with PAA-GO for 48 hours remained fully viable, indicating that the nanocarrier does not have any toxic effect toward GL261 cells (Figure 7). In contrast, free BCNU and PAA-GO-BCNU were toxic to GL261 cells in a concentration-dependent manner. The $\mathrm{IC}_{50}$ (ie, the concentration required for $50 \%$ inhibition of cellular growth after incubation for 48 hours) of PAA-GO-BCNU was $18.2 \mu \mathrm{g} / \mathrm{mL}$, which was only $23 \%$ that of free BCNU $(78.5 \mu \mathrm{g} / \mathrm{mL}$, Figure $8 \mathrm{~A}$ ). The anticancer activity shown by BCNU is related to its ability to induce DNA interstrand cross-linking as the 2-chloroethanediazonium ions produced by $\mathrm{BCNU}$ binds to guanine residues of DNA at the $O^{6}$-position to form $O^{6}$-(2-chloroethyl)deoxyguanosine and kill the cells. ${ }^{36}$ Hence, the extent of DNA cross-linking was also used to determine the anticancer efficacy of PAA-GO-BCNU. The occurrence of interstrand cross-linking was about $15.5 \%$ after treatment with $40 \mu \mathrm{g} / \mathrm{mL}$ of free BCNU for 12 hours but increased approximately 1.3 -fold to $19.6 \%$ using the same concentration of PAA-GO-BCNU (Figure 8B). This higher level of interstrand cross-linking correlated with increased cytotoxicity.

Fluorescence microscopy confirmed that cytotoxicity was proportional to the dose of free $\mathrm{BCNU}$ and $\mathrm{PAA}-\mathrm{GO}-$ BCNU (Figure 9). The live cells (green color) decreased with increasing concentration of PAA-GO-BCNU. Presumably, the increased cytotoxicity of PAA-GO-BCNU toward GL261 cells was most likely via a combined effect from the increased intracellular drug concentration via efficient endocytosis of PAA-GO-BCNU and the improved drug thermal stability resulting from a decreased drug hydrolysis rate.

\section{Conclusion}

In summary, we have successfully functionalized nanosized GO with PAA to form PAA-GO which serves as an efficient nanocarrier for BCNU by covalent binding. Under the optimum condition, the nanocarrier could carry $198 \mu \mathrm{g}$ $\mathrm{BCNU} / \mathrm{mg}$ PAA-GO with $70 \%$ retention of drug activity. This nanocarrier was also demonstrated to prolong the halflife of BCNU significantly from 19 hours to 43 hours. The efficient uptake of PAA-GO by cancer cells can increase the intracellular concentration of BCNU covalently bound to the nanocarrier. The in vitro anticancer efficacy of PAAGO-BCNU was demonstrated by a $30 \%$ increase in DNA interstrand cross-linking and a $77 \%$ decrease in the $\mathrm{IC}_{50}$ value toward GL261 cancer cells compared with the same dosage of free-BCNU.

\section{Acknowledgments}

The authors are grateful for the financial support of the National Science Council of Taiwan, ROC, Chang Gung Memorial Hospital, and the boost program of Chang Gung Memorial Hospital and National Tsing Hua University is also gratefully acknowledged.

\section{Disclosure}

The authors report no conflicts of interest in this work.

\section{References}

1. Ferrari M. Cancer nanotechnology: opportunities and challenges. Nat Rev Cancer. 2005;5:161-171.

2. Pan DJ, Turner JL, Wooley KL. Shell cross-linked nanoparticles designed to target angiogenic blood vessels via $\alpha v \beta 3$ receptor-ligand interactions. Macromolecules. 2004;37:7109-7115. 
3. Chithrani BD, Ghazani AA, Chan WCW. Determining the size and shape dependence of gold nanoparticle uptake into mammalian cells. Nano Lett. 2006;6:662-668.

4. Endo M, Strano MS, Ajayan PM. Potential Applications of Carbon Nanotubes. Topics in Applied Physics. Berlin/Heidelberg, Germany: Springer-Verlag; 2008.

5. Moses M, Brem H, Langer R. Advancing the field of drug delivery: taking aim at cancer. Cancer Cell. 2003;4:337-341.

6. Debbage P. Targeted drugs and nanomedicine: present and future. Curr Pharm Des. 2009;15:153-172.

7. Novoselov KS, Geim AK, Morozov SV, et al. Electric field effect in atomically thin carbon films. Science. 2004;306:666-669.

8. Compton OC, Nguyen ST. Graphene oxide, highly reduced graphene oxide, and graphene: versatile building blocks for carbon-based materials. Small. 2010;6:711-723.

9. Liu Z, Robinson JT, Sun X, Dai H. PEGylated nanographene oxide for delivery of water-insoluble cancer drugs. J Am Chem Soc. 2008;130:10876-10877.

10. Lu CH, Yang HH, Zhu CL, Chen X, Chen GN. A graphene platform for sensing biomolecules. Angew Chem Int Ed. 2009;48:4785-4787.

11. Lu CH, Zhu CL, Li J, Liu JJ, Chen X, Yang HH. Using graphene to protect DNA from cleavage during cellular delivery. Chem Commun. 2010;46:3116-3118.

12. Yang X, Wang Y, Huang X, et al. Multi-functionalized graphene oxide based anticancer drug-carrier with dual-targeting function and pH-sensitivity. J Mater Chem. 2011;21:3448-3454.

13. Yang K, Zhang S, Zhang G, Sun X, Lee ST, Liu Z. Graphene in mice: ultrahigh in vivo tumor uptake and efficient photothermal therapy. Nano Lett. 2010;10:3318-3323.

14. Sun X, Liu Z, Welsher K, et al. Nano-graphene oxide for cellular imaging and drug delivery. Nano Res. 2008;1:203-212.

15. Holland EC. Glioblastoma multiforme: The terminator. Proc Natl Acad Sci U S A. 2000;97:6242-6244.

16. Tait MJ, Petrik V, Loosemore A, Bell BA, Papadopoulos MC. Survival of patients with glioblastoma multiforme has not improved between 1993 and 2004: analysis of 625 cases. Br J Neurosurg. 2007;21:496-500.

17. Kornblith PL, Walker M. Chemotherapy for malignant gliomas. J Neurosurg. 1988;68:1-17.

18. Rhines LD, Sampath P, Dolan ME, Tyler BM, Brem H, Weingart J. $\mathrm{O}^{6}$-Benzylguanine potentiates the antitumor effect of locally delivered carmustine against an intracranial rat glioma. Cancer Res. 2000;60: 6307-6310.

19. Wang Y, Shi ZQ, Huang Y, et al. Supercapacitor devices based on graphene materials. J Phys Chem C. 2009;113:13103-13107.

20. Sahoo NG, Bao H, Pan Y, et al. Functionalized carbon nanomaterials as nanocarriers for loading and delivery of a poorly water-soluble anticancer drug: a comparative study. Chem Commun. 2011;47:5235-5237.
21. Zhang L, Xia J, Zhao Q, Liu L, Zhang Z. Functional graphene oxide as a nanocarrier for controlled loading and targeted delivery of mixed anticancer drugs. Small. 2010;6:537-544.

22. Zhang L, Lu Z, Zhao Q, Huang J, Shen H, Zhang Z. Enhanced chemotherapy efficacy by sequential delivery of sirna and anticancer drugs using PEI-grafted graphene oxide. Small. 2011;7:460-464.

23. Bao H, Pan Y, Ping Y, et al. Chitosan functionalized graphene oxide as a nanocarrier for drug and gene delivery. Small. 2011;7:1569-1578.

24. Leitner VM, Walker GF, Schnurch AB. Thiolated polymers: evidence for the formation of disulphide bonds with mucus glycoproteins. Eur J Pharm Biopharm. 2003;56:207-214.

25. Hummers WS, Offeman RE. Preparation of graphitic oxide. JAm Chem Soc. 1958;80:1339-1345.

26. Yang HW, Hua MY, Liu HL, et al. Self-protecting core-shell magnetic nanoparticles for targeted, traceable, long half-life delivery of BCNU to gliomas. Biomaterials. 2011;32:6523-6532.

27. Whelpton R, Watkins G, Curry SH. Bratton-Marshall and liquidchromatographic methods compared for determination of sulfamethazine acetylator status. Clin Chem. 1981;27:1911-1914.

28. Hua MY, Liu HL, Yang HW, et al. The effectiveness of a magnetic nanoparticle-based delivery system for BCNU in the treatment of gliomas. Biomaterials. 2011;32:516-527.

29. Kawamitsu HU, Lawson TA, Gwilt PR. In vitro pharmacokinetics and pharmacodynamics of 1,3-bis(2-chloroethyl)-1-nitrosourea (BCNU). Biochem Pharmacol. 2002;63:1209-1218.

30. Wang S, Jiang SP, Wang X. Microwave-assisted one-pot synthesis of metal/metal oxide nanoparticles on graphene and their electrochemical applications. Electrochim Acta. 2011;56:3338-3344.

31. Leadley SR, Watts JF. The use of XPS to examine the interaction of poly(acrylic acid) with oxidized metal substrates. J Electron Spectrosc. 1997;85:107-121.

32. Inoue C, Kaneda Y, Aida M, Endo K. Simulation of XPS of poly(vinyl alcohol), poly(acrylic acid), poly(vinyl acetate), and poly(methyl methacrylate) polymers by an ab initio mo method using the model molecules. Polym J. 1995;27:300-309.

33. Xu X, Chen X, Xu X, et al. BCNU-loaded PEG-PLLA ultrafine fibers and their in vitro antitumor activity against glioma C6 cells. J Control Release. 2006;114:307-316.

34. Lee DG, Ponvel KM, Kim M, Hwang S, Ahn IS, Lee CH. Immobilization of lipase on hydrophobic nano-sized magnetite particles. J Mol Catal B Enzym. 2009;57:62-66.

35. Murray RK, Granner DK, editors. Membranes: Structure, Assembly, and Function. Harper's Biochemistry. 25th ed. New York, NY: McGraw-Hill; 2000.

36. Rajski SR, Williams RM. DNA cross-linking agents as antitumor drugs. Chem Rev. 1998;98:2723-2795
International Journal of Nanomedicine

\section{Publish your work in this journal}

The International Journal of Nanomedicine is an international, peerreviewed journal focusing on the application of nanotechnology in diagnostics, therapeutics, and drug delivery systems throughout the biomedical field. This journal is indexed on PubMed Central,

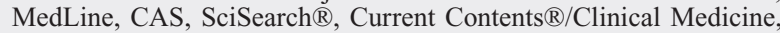

\section{Dovepress}

Journal Citation Reports/Science Edition, EMBase, Scopus and the Elsevier Bibliographic databases. The manuscript management system is completely online and includes a very quick and fair peer-review system, which is all easy to use. Visit http://www.dovepress.com/ testimonials.php to read real quotes from published authors. 